\title{
A Definition of Proximal and Distal Tubular Compliance
}

\author{
PRACTICAL AND THEORETICAL IMPLICATIONS
}

\author{
Stanley Cortell, F. John Gennari, Michael Davidman, William H. Bossert, and \\ William B. Schwartz with the technical assistance of Melvin L. Ponte \\ From the Department of Medicine, Tufts University School of Medicine, and \\ the Renal Service of the New England Medical Center Hospital, \\ Boston, Massachusetts 02111
}

\begin{abstract}
A B S T R A C T Micropuncture studies were carried out in the rat to evaluate the in situ distensibility characteristics of the proximal and distal tubules under a variety of experimental conditions. In the first phase, we determined the response of tubular diameter $(D)$ to changes in tubular pressure $(P)$ induced by partially obstructing single tubules. The response observed under these conditions (i.e., when interstitial pressure is presumed to be constant) has been defined as the compliance of the tubule. Over the range of tubular pressures studied (10$35 \mathrm{~mm} \mathrm{Hg}$ for the proximal tubule, $5-25 \mathrm{~mm} \mathrm{Hg}$ for the distal tubule) the compliance characteristics of the proximal and distal tubule were found to be markedly different; the proximal tubular pressure-diameter relationship was linear, $\Delta D / \Delta P=0.45 \mu \mathrm{m} / \mathrm{mm} \mathrm{Hg}$, whereas the distal pressure-diameter relationship was curvilinear, $\Delta D / \Delta P=e^{-0.1 \times P+2.2}$.
\end{abstract}

In the second phase we used the compliance data to construct a series of theoretical pressure-diameter curves that define the response of the tubule to increments in interstitial as well as intratubular pressure. These curves indicate that changes in distal diameter should provide a sensitive index of a rise in interstitial pressure under conditions in which the transtubular pressure gradient is increased by a small amount, but that proximal diameter should provide a more sensitive index of changes in interstitial pressure when the transtubular pressure gradient is increased by a large amount. In

Dr. Cortell is the recipient of a Career Development Award (HE-35,834) National Heart and Lung Institute, National Institutes of Health; Dr. Gennari is the recipient of a Career Development Award (HE-38,908) National Heart and Lung Institute, National Institutes of Health; and Dr. Davidman is the recipient of a Fellowship from the Medical Research Council of Canada.

Received for publication 9 January 1973 and in recised form 17 April 1973. subsequent experiments in which furosemide was administered, we observed that the pressure-diameter relationships for both the proximal and distal tubule were indistinguishable from the compliance curves, a finding consistent with the interpretation that interstitial pressure was not appreciably changed from control. By contrast, when mannitol was administered, both proximal and distal tubular pressure-diameter relationships were significantly altered in a fashion consistent with a large increase in interstitial pressure. Neither with furosemide nor mannitol administration did it appear likely that significant changes in tubular compliance could account for the observed behavior of the tubule.

Finally, we propose that a knowledge of tubular compliance will be useful in exploring the interrelationships between tubular and peritubular pressures, tubular anatomy, and transtubular ionic permeability. Recent studies linking changes in the geometry of lateral intercellular spaces of the tubule to changes in passive ion movement suggest that an investigation of such anatomical-functional correlates should be productive.

\section{INTRODUCTION}

Recent evidence suggesting that intrarenal pressures play an important role in transtubular ion movement has created a renewed interest in the relationship between tubular function and the physical properties and anatomical characterstics of the tubular epithelium $(1,2)$. Although the response of renal tubular structures to changes in pressure has become a matter of considerable interest, little is known about the in situ response of tubular diameter to increases in tubular pressure. In the first phase of the present study we have examined the relationship between tubular diameter and pressure over a wide range of increases in tubular pressure induced 
by partially blocking single tubules. This relationship, which we have defined as tubular compliance, was found to be notably different for the proximal and distal tubules.'

In the second phase of the study, we have used these compliance curves to construct a series of theoretical pressure-diameter relationships defining the anticipated behavior of tubular diameter if interstitial as well as intratubular pressure were increased. These theoretical curves indicate that changes in distal diameter should provide a sensitive indicator of increases in interstitial pressure under conditions in which the transtubular pressure gradient is increased by a small amount, but that proximal diameter should be more sensitive to increases in interstitial pressure under circumstances in which the transtubular pressure gradient is large. Subsequent observations during mannitol and furosemide diuresis, when analyzed within the framework of these theoretical curves, were consistent with the interpretation that interstitial pressure did not increase appreciably during furosemide diuresis but that it increased markedly during mannitol diuresis. The data also strongly suggested that drug-induced changes in compliance could not reasonably be invoked to account for the observed pressure-diameter relationships.

\section{METHODS}

All studies were performed on male Charles River rats $(200-300 \mathrm{~g})$ (Charles River Breeding Laboratories, Inc., Wilmington, Mass.) fed a standard rat diet and allowed water ad lib. After being anesthetized with Inactin 80-90 $\mathrm{mg} / \mathrm{kg}$ intraperitoneally, the animal was prepared for micropuncture as described previously (3), except that the ureter was catheterized with a PE50 rather than a PE10 catheter.

Upon completion of surgical preparation, all animals were required to meet the following criteria, or the experiment was discontinued: (a) Blood pressure greater than $95 \mathrm{~mm}$ $\mathrm{Hg},(b)$ body temperature between $36-38^{\circ} \mathrm{C},(c)$ urine free of gross blood, $(d)$ transit time (by method of Steinhausen [4]) less than $13 \mathrm{~s}$ with dye flowing evenly to all areas of the kidney and with no retention of dye in the tubules.

If the above conditions were met, $25 \mu \mathrm{Ci}$ of $\left[{ }^{14} \mathrm{C}\right]$ inulin was given as a bolus, and $\left[{ }^{14} \mathrm{C}\right]$ inulin was added to the sustaining infusion in sufficient concentration to deliver 25 $\mu \mathrm{Ci} / \mathrm{h}$. After a $30 \mathrm{~min}$ equilibration period, the experimental protocols described below were begun.

\section{Experimental protocols}

The effect of increased intratubular pressure upon tubular diameter was studied in the antidiuretic state in one series of experiments ("partial blockade studies") and in two high flow states in a second series of experiments ("diuretic

${ }^{1}$ As we shall point out later, because the proximal and distal tubules were necessarily studied over a different range of pressures, the difference in compliance observed for these two segments of the nephron does not necessarily imply a difference in their inherent distensibilities. studies"). The two experimental protocols are described in detail below.

Partial blockade studies. In these experiments, increases in intratubular pressure were induced in single proximal or distal tubules by means of a castor oil block. The infusion begun during surgical preparation (5\% mannitol in $0.5 \%$ saline) was discontinued when surgery was completed, and an infusion of $0.5 \%$ saline was given at $1.7 \mathrm{~cm}^{3} / \mathrm{h}$ for the remainder of the experiment.

Single tubules were studied in the following manner: Resting intratubular pressure was measured and the pressure-measuring pipette was left in place. Lissamine green dye was then infused into the tubule through this pipette and photographs were taken (the details of the techniques will be described later). After the photographs, the tubular pressure was remeasured. A pipette filled with castor oil was then placed at a more distal site in the same tubule and tubular flow was partially blocked by the insertion of a long oil block. If tubular pressure after partial blockade increased by $5 \mathrm{~mm} \mathrm{Hg}$ or more in the proximal tubule or by $2 \mathrm{~mm} \mathrm{Hg}$ or more in the distal tubule, the same sequence of pressure, picture, and pressure measurement was then repeated.

Data from proximal tubules were not accepted unless the pressure after picture-taking was within $5 \mathrm{~mm} \mathrm{Hg}$ of the pressure measured before pictures were taken; data from distal tubules were not accepted unless the tubular pressures before and after picture taking were within $2 \mathrm{~mm} \mathrm{Hg}$ of each other. These different criteria for pressure changes in the proximal and distal tubule were established on the basis of preliminary studies in which it was noted that during partial blockade, tubular pressure was less stable in the proximal tubule than in the distal tubule. The mean difference between the pressures before and after pictures was $0.25 \mathrm{~mm} \mathrm{Hg}$ for the proximal tubules (in $40 \%$ the repeat pressure was lower, in $55 \%$ the repeat pressure was higher, and in $5 \%$ there was no change), and $-0.18 \mathrm{~mm}$ $\mathrm{Hg}$ for the distal tubule (56\% lower, $38 \%$ higher, $6 \%$ no change).

Diuretic studies. Changes in intratubular pressure were induced by increasing tubular flow by infusion of either mannitol or furosemide. The experiments were divided into three periods: control, low-dose diuresis, and high-dose diuresis. In each period photographs were taken of the kidney surface for diameter measurements. Intratubular pressure measurements were made in at least one proximal and one distal tubule both before and after picture taking and in a total of not less than three proximal and three distal tubules. In the control period, an infusion of $5 \%$ mannitol in $0.5 \%$ saline (begun during neck surgery) was maintained at $1.7 \mathrm{~cm}^{3} / \mathrm{h}$. No notable diuresis was produced by this amount of mannitol as evidenced by a mean control $\mathrm{U} / \mathrm{P}$ inulin ratio of 378 and a mean urine flow rate of 9 $\mu \mathrm{l} / \mathrm{min}$. The two experimental periods for mannitol and furosemide are described below.

Mannitol. In the low-dose diuretic period, $0.8 \mathrm{~cm}^{3}$ of $10 \%$ mannitol was given as a bolus, and a solution of $10 \%$ mannitol in $0.5 \%$ saline was then infused at $9 \mathrm{~cm}^{3} / \mathrm{h}$. In the high-dose diuretic period $1 \mathrm{~cm}^{3}$ of $10 \%$ mannitol was given as a bolus, and the $10 \%$ mannitol solution was then infused at $18 \mathrm{~cm}^{3} / \mathrm{h}$. In order to attain a relatively constant urine flow in each experimental period, a $30 \mathrm{~min}$ equilibration period was allowed before pressure and diameter measurements were made.

Furosemide. In the low-dose diuretic period, $5 \mathrm{mg} / \mathrm{kg}$ of furosemide was administered as a bolus and the drug 

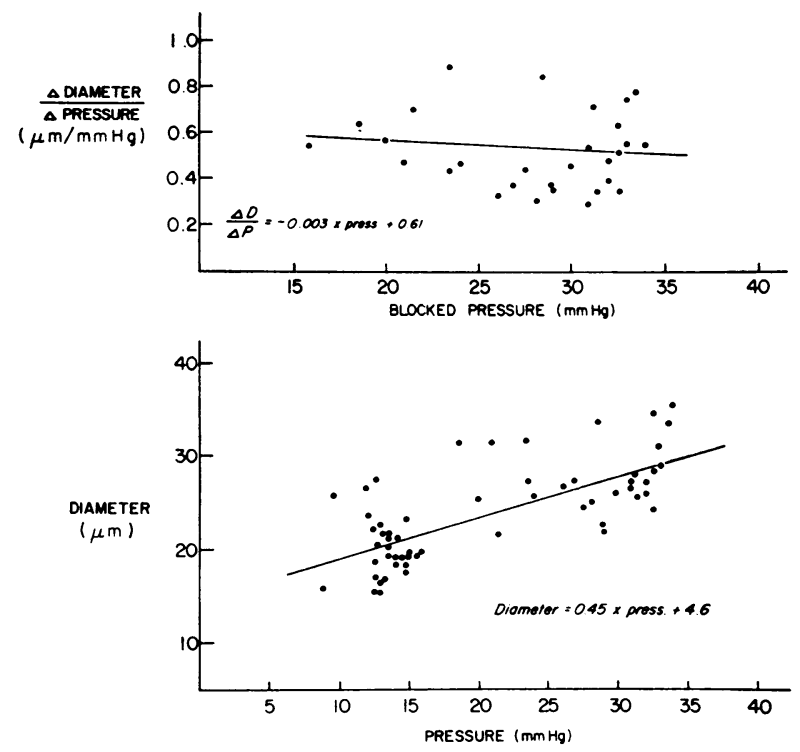

Figure 1 Proximal tubular compliance. In the upper panel, the change in diameter per $\mathrm{mm} \mathrm{Hg}$ change in tubular pressure $(\Delta D / \Delta P)$ is plotted against the absolute tubular pressure during partial blockade. In the lower panel, the absolute diameter is plotted against tubular pressure during control and partial blockade; thus each tubule is represented by two points. The lines drawn through the data points in both panels were calculated by the method of least squares.

then continuously infused in $0.7 \%$ saline at a rate of $5 \mathrm{mg} /$ $\mathrm{kg} / \mathrm{h}$. In the high-dose period $25 \mathrm{mg} / \mathrm{kg}$ of furosemide was given as a bolus, and then continuously infused in $0.7 \%$ saline at a rate of $50 \mathrm{mg} / \mathrm{kg} / \mathrm{h}$. In both experimental periods, a $20 \mathrm{~min}$ equilibration period was allowed before pressure and diameter measurements were made.

During the two diuretic periods (in both the mannitol and furosemide experiments), urinary losses of fluid and electrolyte were quantitatively replaced in order to minimize changes in extracellular fluid volume. To approximate the sodium content of urinary losses induced by each agent, $0.5 \%$ saline was used for replacement in the mannitol experiments and $0.7 \%$ saline in the furosemide experiments. Data from an animal were accepted only if the fluid balance (total infusion minus urine flow for the two kidneys) ${ }^{2}$ for the entire 4-5 h experiment was $0-6 \mathrm{~cm}^{3}$ positive. This slightly positive fluid balance was allowed to account for insensible losses. The mean cumulative fluid balance was $+4 \mathrm{~cm}^{3}$ for the furosemide experiments and $+3 \mathrm{~cm}^{3}$ for the mannitol experiments.

\section{Techniques and analytical methods}

Proximal and distal tubular pressures were measured by a modification of the technique of Gottschalk and Mylle (5)

${ }^{2}$ Urine flow of the two kidneys was estimated by doubling measured single-kidney flow. To examine whether this extrapolation was justified, urine flow rate, $U / P$ inulin ratio, and GFR were measured for both the micropunctured kidney and the control kidney in four rats (two with mannitol and two with furosemide): no difference between the two kidneys was observed. described previously from our laboratory (3). $\left[{ }^{14} \mathrm{C}\right]$ Inulin activity in plasma and urine was measured as previously described (6).

Tubular diameter measurements. Photographs of the kidney surface were taken on high-speed Ektachrome film at $\times 40$ magnification utilizing one ocular of a Leitz stereo microscope (E. Leitz, Inc., Rockleigh, N. J.). The field was illuminated with a synchronized Zeiss Ukatron 60 Flash attachment (Carl Zeiss, Inc., New York). The photographs for diameter measurement were taken after intratubular lissamine green injection in the partial blockade experiments, and after intravenous lissamine green injection in the diuretic experiments.

Intratubular method (partial blockade studies). A $1 \%$ lissamine green solution was infused into individual tubules by increasing the pressure in the pipette above the intratubular pressure until dye began to flow into the tubule. To minimize any intratubular pressure changes that might occur with lissamine green injection, the pipette pressure was then rapidly lowered and the pictures were taken at a time when the pipette pressure was approximately equal to the intratubular pressures. Photographs were not used for diameter measurements unless the pressure in the pipette at the time of picture-taking was within $5 \mathrm{~mm} \mathrm{Hg}$ of the intratubular pressure. In actuality, the mean pipette pressure was $0.9 \mathrm{~mm} \mathrm{Hg}$ above the intratubular pressure for the proximal tubule and $0.5 \mathrm{~mm} \mathrm{Hg}$ above the intratubular pressure for the distal tubule.

Intravenous method (diurctic studies). After the intravenous injection of $0.05 \mathrm{~cm}^{3}$ of a $10 \%$ lissamine green solution, photographs were taken of dye-filled proximal and distal tubules. Since surface distal tubules are much less numerous than proximal, an attempt was made to photograph several different areas of the kidney surface after each injection. If the same distal tubule was photographed more than once, it was only measured in the first frame.

Technique of measurement. The photographs were projected on a white cardboard screen with the projector kept a standard distance from the screen. Each roll of film included a photograph of a stage micrometer taken at the same magnification as the photographs of the kidney surface. All tubular diameters were arbitrarily measured as the width of the intratubular green-dye column. The diameter measurements were carried out by a team of two observers who had no knowledge of which experiment or which experimental period they were measuring. The diameters were measured by one observer, using calipers; the second observer recorded the caliper width. A $50 \mu \mathrm{m}$ segment of the stage micrometer was also measured and recorded for conversion of the diameter measurements to micrometers. One-quarter of all the diameters were remeasured by a team of two observers brought in from outside the laboratory. Their measurements were compared with the first group's measurements and no significant differences were noted.

Proximal tubules were measured in the following manner: In the intravenous injection series, each clearly-focused, dye-filled tubular loop was measured if the borders were roughly parallel for at least 1 diam width. In the intratubular injection series, a measurement was made in each segment of the dye-filled loop if its borders were roughly parallel. Loop segments containing a pipette were not measured.

Distal tubules were measured in the following manner: In the intravenous injection series, the widths of dye-filled tubules were measured in the same manner as were the 
proximal tubules except that in those tubules with irregular borders, measurements were made both at the narrowest and widest places where the borders were roughly parallel; these two measurements were averaged. In the intratubular injection series, each loop was measured in two to three places, including the narrowest and the widest areas. No measurements were made within 1 diam width of the pipettes.

\section{RESULTS}

\section{Effect of partial blockade on pressure and diameter in individual tubules}

Proximal tubule. The effect of increased tubular pressure upon tubular diameter was studied by means of partial blockade in individual proximal tubules in 17 rats. The mean tubular pressure was $13.1 \pm 0.3 \mathrm{~mm} \mathrm{Hg}$ before blockade. In 30 out of 34 successfully blocked tubules, intratubular pressure increased by $5 \mathrm{~mm} \mathrm{Hg}$ or more. For each tubule fulfilling this criterion for increased pressure (see Methods), the change in tubular diameter per $\mathrm{mm} \mathrm{Hg}$ change in tubular pressure $(\Delta D / \Delta P)$ was plotted against the absolute tubular pressure during partial blockade (upper portion of Fig. 1)..$^{3}$ The slope of the calculated regression line drawn through these data points was not significantly " different from zero, indicating that the relationship between tubular diameter and pressure (lower portion of Fig. 1) was linear over the range of pressures studied. The mean value for $\Delta D / \Delta P$ was $0.51 \pm 0.03 \mu \mathrm{m} / \mathrm{mm} \mathrm{Hg}$ (Fig. 3 ). The control and "partial blockade" values for tubular diameter are plotted against tubular pressure in an unpaired fashion in the lower portion of Fig. 1. The slope of the calculated linear regression for these data, $0.45 \mu \mathrm{m} / \mathrm{mm}$ $\mathrm{Hg}$, was significantly greater than zero.

Distal tubule. The effect of increased distal tubular pressure upon tubular diameter was studied by means of partial blockade in individual distal tubules in 16 rats. The mean tubular pressure was $6.7 \pm 0.3 \mathrm{~mm} \mathrm{Hg}$ before blockade. In all 23 tubules successfully blocked, intratubular pressure increased by the required 2 or more $\mathrm{mm} \mathrm{Hg}$ (see Methods). For each tubule studied, the change in tubular diameter per $\mathrm{mm} \mathrm{Hg}$ change in tubular pressure $(\Delta D / \Delta P)$ was plotted against the absolute pressure during partial blockade (upper portion of Fig. 2). As can be seen from inspection of this figure, in contrast to the behavior of the proximal tubule, $\Delta D / \Delta P$ for the distal tubule decreased as tubular pressure was increased by partial blockade, indicating a nonlinear relationship between tubular pressure and diameter.

\footnotetext{
${ }^{3}$ In one tubule in which intratubular pressure increased by $5 \mathrm{~mm} \mathrm{Hg}$, a negative value was obtained for $\Delta D / \Delta P$; this value was found to be an outlier variant $(P<0.01)$ and was discarded (7).

"Throughout this paper the term "significant" will indicate a $P$ value of less than 0.01 .
}

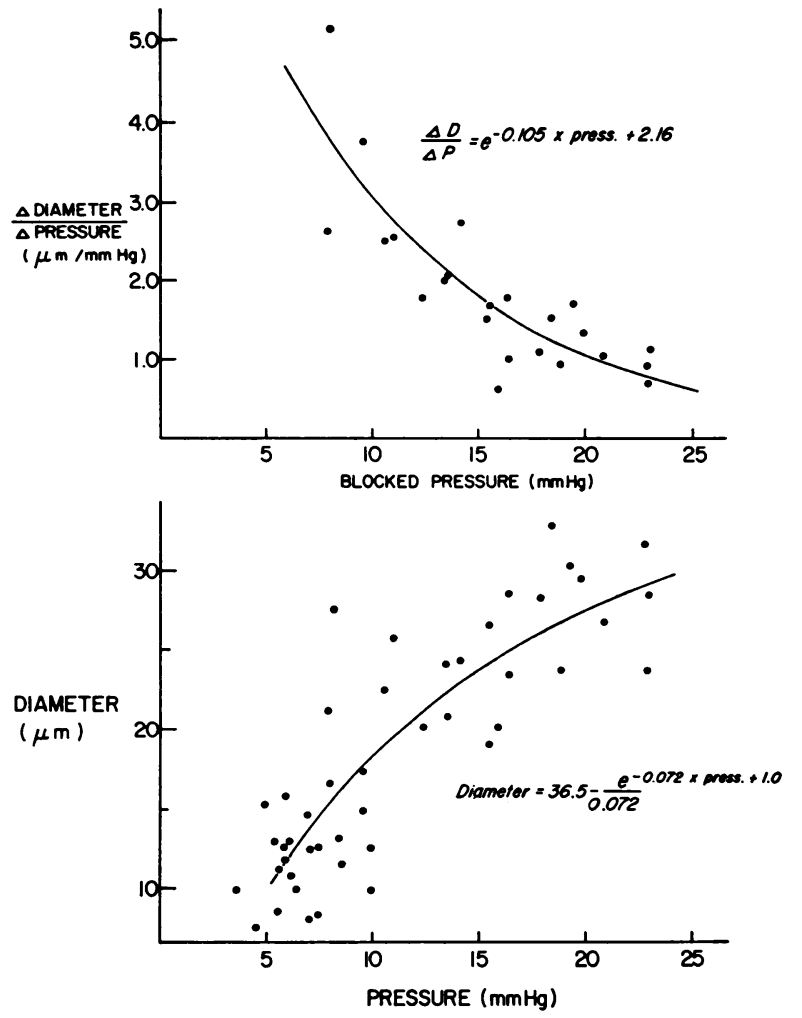

FigURE 2 Distal tubular compliance. In the upper panel, the change in diameter per $\mathrm{mm} \mathrm{Hg}$ change in tubular pressure $(\Delta D / \Delta P)$ is plotted against the absolute tubular pressure during partial blockade. In the lower panel, the absolute diameter is plotted against tubular pressure during control and partial blockade; thus each tubule is represented by two points. The curves drawn through the data points in both panels were calculated by means of a computer-based nonlinear least-squares search.

In the lower portion of Fig. 2, both the control and "partial blockade" values for tubular diameter are plotted against tubular pressure. From this representation of the data, it can be seen that small increases in intratubular pressure above control were associated with large changes in tubular diameter and that progressively larger changes in pressure were associated with ever smaller additional increments in tubular diameter. On the basis of this pattern of diameter change, we have chosen an exponential function defining an asymptotic regression to represent the relationship between distal tubular diameter and pressure. It should be pointed out, however, that the shape of the resulting curve will be closely similar whether an exponential function or a quadratic or higher-power polynomial function is used to represent the data. In the equation for the exponential function, $D=C-1 / A \times e^{-A \times P+B}$, the values for $A, B$, and $C$ were calculated for our data points using a computer-based nonlinear least-squares search. This expo- 


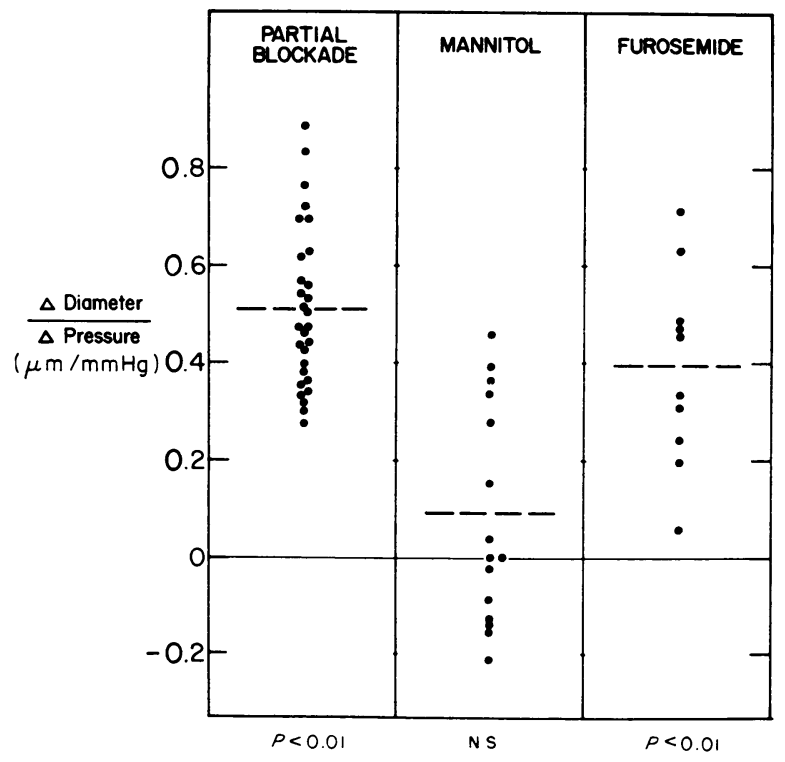

Figure 3 Changes in proximal tubular diameter per $\mathrm{mm}$ $\mathrm{Hg}$ increase in tubular pressure $(\Delta D / \Delta P)$ during partial blockade of single nephrons (left-hand panel), mannitol diuresis (center panel), and furosemide diuresis (right-hand panel). Each point in the figure represents the change in tubular diameter divided by the corresponding change in tubular pressure for each experimental observation in which tubular pressure increased by 5 or more $\mathrm{mm} \mathrm{Hg}$. The dashed line in each panel indicates the mean value for each group of observations. Note that the mean values for $\Delta D / \Delta P$ in the partial blockade and furosemide studies are significantly greater than zero, whereas the mean value in the mannitol study is not significantly different from zero.

nential function provided the basis for using the function, $\Delta D / \Delta P=e^{-A \times P+B}$, to describe the relationship between $\Delta D / \Delta P$ and the blocked pressure in the upper portion of the figure. The values for $A$ and $B$ in the latter equation were also calculated from our data using the nonlinear least-squares search. The resulting equation, $\Delta D / \Delta P=e^{-0.105 \times P+2.16}$, accounted for $71 \%$ of the total variation in $\Delta D / \Delta P$.

\section{Effect of mannitol-induced diuresis on tubular pressure and diameter}

Proximal tubule. Proximal tubular pressure increased from a mean value of $12.1 \mathrm{~mm} \mathrm{Hg}$ in the control period to a mean value of $19.4 \mathrm{~mm} \mathrm{Hg}$ in the low-dose period and rose further to $25.4 \mathrm{~mm} \mathrm{Hg}$ in the high-dose period. In 15 determinations of proximal tubular diameter and pressure in 9 rats, proximal tubular pressure increased by 5 or more $\mathrm{mm} \mathrm{Hg}$. For these 15 determinations, as in the case of the proximal tubule in the partial blockade studies, $\Delta D / \Delta P$ did not vary significantly as a function of tubular pressure. The values for $\Delta D / \Delta P$ during mannitol diuresis are shown in the center panel of Fig. 3. The mean value, $0.10 \mu \mathrm{m} / \mathrm{mm} \mathrm{Hg}$, was significantly less than the mean for $\Delta D / \Delta P$ observed for the proximal tubule in the partial blockade studies (left-hand panel of Fig. 3), a finding consistent with the interpretation that interstitial pressure increased during mannitol diuresis.

Distal tubule. Distal tubular pressure increased from a mean value of $6.8 \mathrm{~mm} \mathrm{Hg}$ in the control period to a mean value of $14.8 \mathrm{~mm} \mathrm{Hg}$ in the low-dose period and to $20.6 \mathrm{~mm} \mathrm{Hg}$ in the high-dose period. In 18 determinations of distal tubular pressure and diameter in 9 rats, distal tubular pressure increased by 2 or more $\mathrm{mm} \mathrm{Hg}$ above the control value. The mean value for $\Delta D / \Delta P$ during mannitol diuresis, $0.99 \pm 0.09$ $\mu \mathrm{m} / \mathrm{mm} \mathrm{Hg}$, was significantly less than the mean value for the distal tubule during partial blockade, $1.85 \pm 0.2$ $\mu \mathrm{m} / \mathrm{mm} \mathrm{Hg}$. In the left-hand panel of Fig. $4, \Delta D / \Delta P$ is plotted against the absolute pressure during mannitol diuresis. The curve defining this relationship was, when tested by covariance analysis, significantly different from the curve defining the relationship during partial blockade (dashed line); this difference was present regardless of whether a linear, quadratic, or exponential function was used to define the partial blockade curve. ${ }^{5}$ This difference in response is consistent with the interpretation that interstitial pressure increased during mannitol diuresis. It should be noted that distal diameter during mannitol diuresis differed most strikingly from that observed in the partial blockade studies when tubular pressure was increased by a small or moderate amount. At levels of tubular pressure above $20 \mathrm{~mm} \mathrm{Hg}$, $\Delta D / \Delta P$ in the two studies converged.

\section{Effect of furosemide-induced diuresis on tubular pressure and diameter}

Proximal tubule. Proximal tubular pressure increased from a mean value of $13.3 \mathrm{~mm} \mathrm{Hg}$ in the control period to a mean value of $18.5 \mathrm{~mm} \mathrm{Hg}$ in the low-dose period but did not increase further in the high-dose period. In 10 determinations of proximal

${ }^{5}$ As noted earlier, on the basis of the pattern of diameter change an exponential function was chosen to represent the relationship between distal tubular diameter and pressure in the partial blockade studies. However, if instead one utilizes a quadratic or cubic function to describe this relationship, the same statistical difference between mannitol and the partial blockade studies is noted. This conclusion was arrived at on the basis of the following analysis. For both the quadratic and the cubic functions, the appropriate derivative functions were obtained and fitted to the data relating $\Delta D / \Delta P$ to tubular pressure (left-hand panel of Fig. 4) by a least-squares search. The resultant relationship, represented by a linear equation when the diameter-pressure curve was described by a quadratic function and by a quadratic function when the diameter-pressure relationship was described by a cubic function, was then compared with the mannitol data by means of a covariance analysis. 


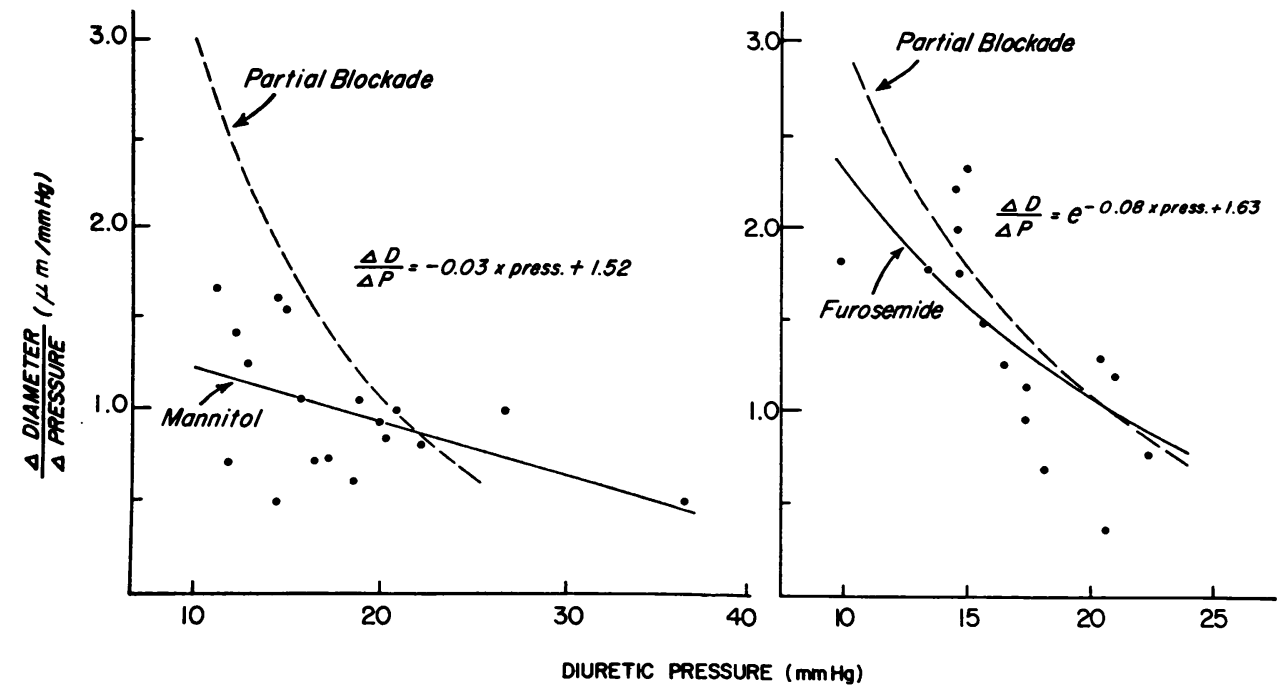

Figure 4 Changes in distal tubular diameter per $\mathrm{mm} \mathrm{Hg}$ increase in tubular pressure $(\Delta D /$ $\Delta P$ ) during mannitol diuresis (left-hand panel) and during furosemide diuresis (right-hand panel). In each panel $\Delta D / \Delta P$ is plotted against the absolute pressure during diuresis for each experimental observation in which tubular pressure increased by 2 or more $\mathrm{mm} \mathrm{Hg}$. The solid lines drawn through the data points in each panel were calculated by means of a least-squares search. The dashed line in each panel represents the relationship between $\Delta D / \Delta P$ and absolute pressure during the partial blockade experiments. See text for an explanation of the form of equation used to define the relationship during furosemide diuresis.

tubular diameter and pressure in 9 rats, proximal tubular pressure increased by 5 or more $\mathrm{mm} \mathrm{Hg}$. For these 10 determinations, as in the case of the proximal tubule in the partial blockade experiments, $\Delta D / \Delta P$ did not vary significantly as a function of tubular pressure. The values for $\Delta D / \Delta P$ during furosemide diuresis are shown in the right-hand panel of Fig. 3. The mean value, 0.39 $\mu \mathrm{m} / \mathrm{mm} \mathrm{Hg}$, was not significantly different from the mean value for $\Delta D / \Delta P$ observed for the proximal tubule in the partial blockade studies (left-hand panel of Fig. 3 ), but was significantly greater than that observed during mannitol diuresis (center panel).

Distal tubule. Distal tubular pressure increased from a mean value of $7.6 \mathrm{~mm} \mathrm{Hg}$ in the control period to a mean value of $14.3 \mathrm{~mm} \mathrm{Hg}$ in the low-dose period, but as in the case of the proximal tubule, no further significant increase in pressure occurred in the high-dose period. In 15 determinations of distal tubular pressure and diameter in 9 rats, distal tubular pressure increased by 2 or more $\mathrm{mm} \mathrm{Hg}$ above the control value. For these 15 determinations the mean value for $\Delta D / \Delta P(1.39 \pm 0.15 \mu \mathrm{m} / \mathrm{mm} \mathrm{Hg})$ was not significantly different from the mean value for the distal tubule in the partial blockade studies. In the righthand panel of Fig. 4, $\Delta D / \Delta P$ is plotted against the absolute pressure during furosemide diuresis. The curve defining this relationship, using either a linear or exponential function, was not significantly different from the curve defining the relationship in the partial blockade studies (dashed line). These findings indicate that the response of distal tubular diameter during furosemide diuresis is indistinguishable from that observed in the partial blockade studies; we have therefore used an exponential function of the same type as described earlier for the partial blockade experiments to define the relationship during furosemide diuresis.

It should also be noted that the curve defining the relationship during furosemide diuresis was, when tested by covariance analysis, significantly different from that observed during mannitol diuresis (left-hand panel of Fig. 4).

Pressure gradient across the loop of Henle during mannitol and furosemide diuresis

Fig. 5 presents the relationship between the pressure gradient across the loop of Henle (proximal tubular pressure minus distal tubular pressure) and distal tubular pressure before and during mannitol diuresis (lefthand panel), and before and during furosemide diuresis (right-hand panel). During mannitol diuresis the slope of the calculated linear regression was not significantly different from zero, indicating that the pressure gradient did not decrease significantly as distal tubular pressure increased. In contrast, the pressure gradient decreased significantly after furosemide administration, falling 


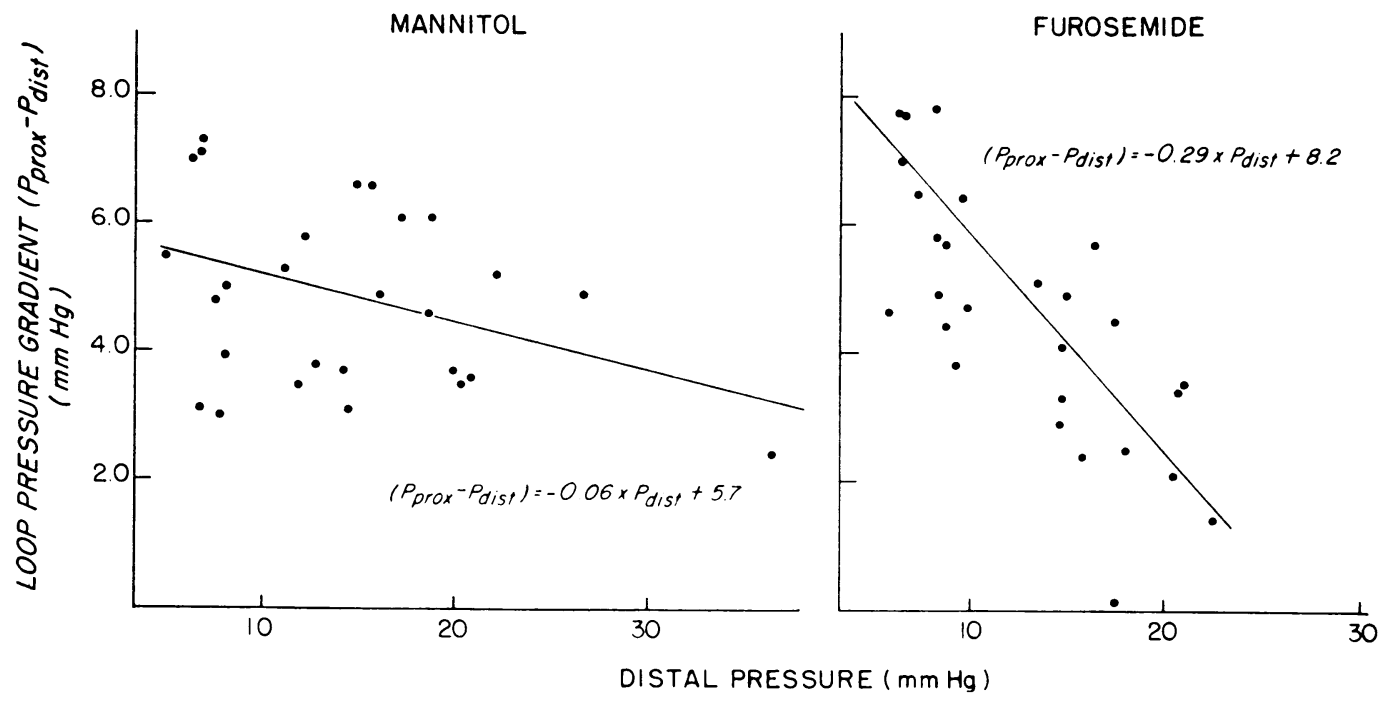

FIgure 5 Pressure gradient across the loop of Henle during mannitol diuresis and furosemide diuresis. For each experimental period (control, low dose, high dose) in each rat, the difference between the proximal and distal tubular pressure is plotted on the ordinate and the distal tubular pressure is plotted on the abscissa. The lines drawn through the data points were calculated by the method of least squares.

from 7 to $2.5 \mathrm{~mm} \mathrm{Hg}$ when the distal tubular pressure rose to $20 \mathrm{~mm} \mathrm{Hg}$.

\section{Urine flow rate and GFR}

In the partial blockade experiments, mean urine flow was $8 \pm 0.8 \mu \mathrm{l} / \mathrm{min}$, mean $\mathrm{U} / \mathrm{P}$ inulin ratio was $528 \pm 27$, and the mean GFR was $12.0 \pm 0.3 \mathrm{~cm}^{3} / \mathrm{min} / \mathrm{kg}$ for two kidneys. In the control period of the mannitol and furosemide experiments, mean urine flow was $9 \pm 0.6$ $\mu \mathrm{l} / \mathrm{min}$, mean $\mathrm{U} / \mathrm{P}$ inulin ratio was $378 \pm 31$, and the mean GFR was $13.7 \pm 0.6 \mathrm{~cm}^{3} / \mathrm{min} / \mathrm{kg}$ for two kidneys. During mannitol diuresis, the mean urine flow rate after equilibration was $178 \mu \mathrm{l} / \mathrm{min}$ in the low-dose period and increased significantly to $497 \mu \mathrm{l} / \mathrm{min}$ in the high-dose period. During furosemide diuresis, the mean urine flow rate was $325 \mu \mathrm{l} / \mathrm{min}$ in the low-dose period and increased significantly to $562 \mu \mathrm{l} / \mathrm{min}$ in the high-dose period. No significant changes in GFR were noted after the administration of either agent. It is noteworthy that during mannitol diuresis, distal pressure increased significantly as urine flow increased, whereas during furosemide diuresis no increase in distal pressure was noted after an increase in urine flow of similar magnitude. Expressed quantitatively, distal tubular pressure increased by $0.032 \mathrm{~mm} \mathrm{Hg} / \mu \mathrm{l}$ increase in urine flow $(P<0.01)$ during mannitol diuresis whereas no significant correlation was noted between distal pressure and urine flow during furosemide diuresis.

\section{DISCUSSION}

The present study has characterized the response of tubular diameter when tubular pressure was varied widely by partially blocking single tubules in situ, that is, under conditions of presumed constancy of interstitial pressure. We have defined the slope of the resultant relationship between tubular pressure and diameter $(\Delta D / \Delta P)$ as the compliance of the tubule. As illustrated in Figs. 1 and 2, the compliance characteristics of the proximal and distal tubules were markedly different. Proximal tubular diameter increased as a linear function of the increase in tubular pressure, $\Delta D / \Delta P=$ 0.45 . In contrast, the distal tubular pressure-diameter relationship was curvilinear, tubular diameter virtually doubling after a $5 \mathrm{~mm} \mathrm{Hg}$ increase in tubular pressure and then increasing by ever smaller increments with progressively larger increases in tubular pressure. ${ }^{6}$

The compliance characteristics of the distal tubule provide strong evidence that in the antidiuretic state interstitial pressure is less than distal tubular pressure.

\footnotetext{
"It should be noted that the difference in proximal and distal tubular compliance after small increases in the transtubular pressure gradient (e.g. $5 \mathrm{~mm} \mathrm{Hg}$ ) does not necessarily imply a difference in inherent tubular distensibility. If, as seems highly probable, interstitial pressure is approximately $6 \mathrm{~mm} \mathrm{Hg}$ in the antidiuretic state (see subsequent discussion in text), it follows that the proximal transtubular pressure gradient before blockade $(6 \mathrm{~mm} \mathrm{Hg})$ is already $5 \mathrm{~mm} \mathrm{Hg}$ greater than the distal transtubular pressure gradient. Thus, the range of transtubular pressures over which the largest change in distal diameter occurred, i.e., 1-6 $\mathrm{mm} \mathrm{Hg}$, cannot be examined for the proximal tubule. In the absence of such observations for the proximal tubule one cannot decide whether or not the inherent distensibility characteristics of the two segments of the nephron are different.
} 
i.e., less than $7 \mathrm{~mm} \mathrm{Hg}$. Given that the distal tubule dilates so readily after a small increase in the transtubular pressure gradient, it seems extremely unlikely that it would become rigid (i.e., not collapse) if the pressure outside were greater than the pressure inside. It is therefore difficult to reach any conclusion but that resting interstitial pressure must be less than distal intratubular pressure. This interpretation is consistent with recent estimates based upon subcapsular measurements of hydrostatic pressure that indicate a value for resting interstitial pressure of $6 \mathrm{~mm} \mathrm{Hg}$ (8).

The compliance characteristics of the distal tubule also suggest that in certain settings distal diameter should be highly sensitve to changes in interstitial pressure. The predicted effect of a wide range of theoretical increases in interstitial pressure on the relationship between distal tubular pressure and diameter is illustrated in the lower panel of Fig. 6. The solid line in the panel was taken from the experimental measurement of tubular compliance ${ }^{7}$ and represents the relationship of tubular pressure to diameter when interstitial pressure remains constant. The dashed lines represent the predicted tubular pressure-diameter relationships if interstitial pressure were increasing by a constant percentage of the increase in tubular pressure (as indicated on the right-hand side of the figure).

As would be anticipated from the curvilinear nature of the distal tubular compliance curve, the sensitivity of distal diameter to changes in interstitial pressure is greatest with small changes in the transtubular pressure gradient and decreases markedly as the transtubular pressure gradient increases. Thus, it is apparent that distal diameter should be highly sensitive to changes in interstitial pressure in any setting in which tubular pressure is increased by a small amount. The sensitivity of distal diameter in such a setting is illustrated by a consideration of points $A$ and $A^{\prime}$ in Fig. 6. In this example, in which tubular pressure is assumed to increase by $5 \mathrm{~mm} \mathrm{Hg}$, a detectable shift in tubular diameter $(2 \mu \mathrm{m})$ away from the compliance curve (from point $A$ to point $A^{\prime}$ ) would be predicted to occur if interstitial pressure were increased by as little as $1.5-2$ $\mathrm{mm} \mathrm{Hg}$ above control. With larger increments in tubular pressure, the sensitivity of distal diameter to small increases in interstitial pressure progressively diminishes, as is illustrated by points $B$ and $B^{\prime}$. In this latter example, in which tubular pressure is increased by 10 $\mathrm{mm} \mathrm{Hg}$, a $2 \mu \mathrm{m}$ shift in tubular diameter (from B to $B^{\prime}$ ) would be predicted to occur only after a 4-5 mm $\mathrm{Hg}$ increase in interstitial pressure. With increments

\footnotetext{
${ }^{7}$ The distal tubular compliance curve was computed from the experimental measurements of $\Delta D$ and $\Delta P$ using a computer-based nonlinear least-squares search. The resulting equation is : $\Delta D=15.3-3.3 \times e^{-0.3 \times \Delta P+1.5}$.
}
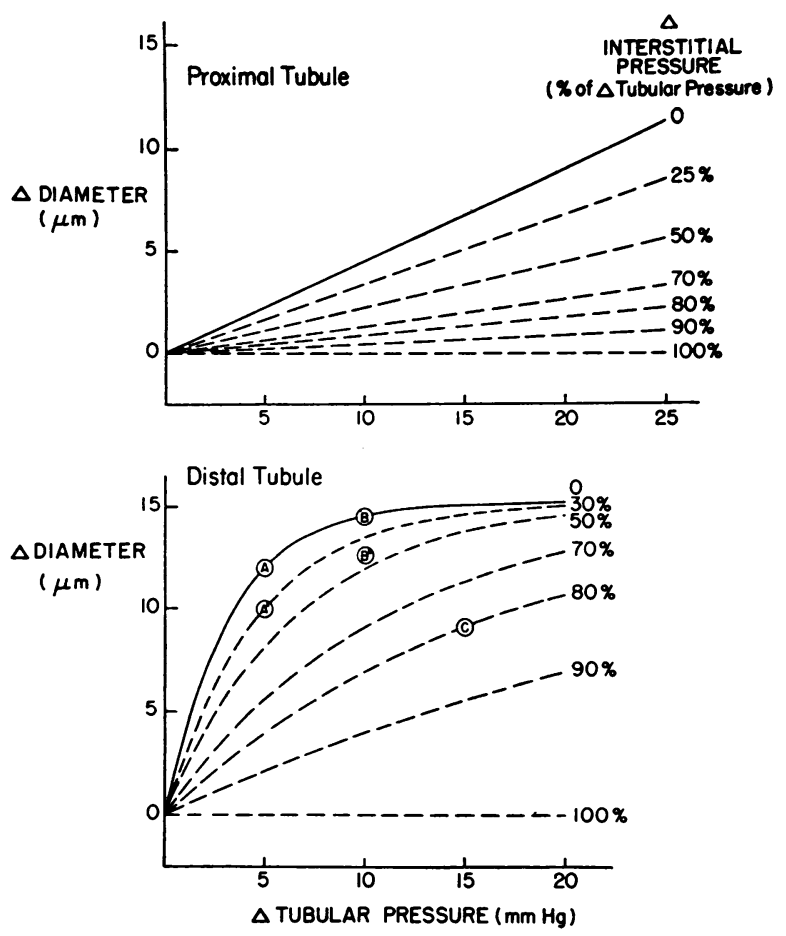

Figcre 6 Predicted effect of a range of theoretical increases in interstitial pressure upon the proximal and distal tubular pressure-diameter relationships. The solid lines in both panels are taken from the experimental measurements of tubular compliance and represent the tubular pressurediameter relationships when interstitial pressure remains constant. The dashed lines represent the predicted tubular pressure-diameter relationships if interstitial pressure were increasing by a constant percentage (as indicated on the right-hand side of the figure) of the increase in tubular pressure. Points $A, A^{\prime}, B, B^{\prime}$, and $C$ in the lower panel are discussed in the text.

in tubular pressure of greater than $10 \mathrm{~mm} \mathrm{Hg}$, it is evident that a detectable shift in distal diameter away from the compliance curve would occur only if interstitial pressure were increased by greater than $50 \%$ of the increase in tubular pressure. By contrast, the proximal tubule (upper panel of Fig. 6) should be useful in detecting relatively small increases in interstitial pressure even when tubular pressure is increased by more than $10 \mathrm{~mm} \mathrm{Hg}$. Because of the linear nature of proximal tubular compliance, a detectable shift in diameter $(2 \mu \mathrm{m})$ away from the compliance curve would be predicted to occur whenever interstitial pressure is increased by $4-5 \mathrm{~mm} \mathrm{Hg}$.

If, on the other hand, interstitial pressure is increased by a large amount, obviously both proximal and distal diameter should readily detect the change. However, distal diameter should provide a more precise estimate of the change when the increase in interstitial pressure is almost as large as the increase in 
TABLE I

Increase in Interstitial P'ressure during Mannitol Diuresis as Estimated from Changes in Tubular

Diameter and Pressure

\begin{tabular}{|c|c|c|c|c|}
\hline \multicolumn{2}{|c|}{$\Delta$ Tubular pressure } & \multirow{2}{*}{$\frac{د D}{\operatorname{Mean} \pm \operatorname{SEM}}$} & \multicolumn{2}{|c|}{$\begin{array}{c}\text { Estimated } \Delta \\
\text { interstitial pressure }\end{array}$} \\
\hline Range & Mean & & Mean & Range* \\
\hline \multicolumn{2}{|c|}{$\begin{array}{c}m m \mathrm{Hg} \\
\text { Distal tubule }\end{array}$} & $\mu m$ & \multicolumn{2}{|c|}{$m m \mathrm{Hg}$} \\
\hline 3-8 & 6 & $6.6 \pm 1.1 \quad(6)$ & 4 & $3-5$ \\
\hline $12-15$ & 13 & $10.9 \pm 1.0(6)$ & 9) & $7-10$ \\
\hline \multicolumn{5}{|c|}{ Proximal tubule } \\
\hline $10-14$ & 12 & $0.6 \pm 0.8$ & 11 & $7-14$ \\
\hline
\end{tabular}

* Range of error obtained by using the mean diameter change \pm 2 SEM to estimate the increase in interstitial pressure.

tubular pressure (i.e., when the net increase in the transtubular pressure gradient is small). Thus, if tubular pressure were increased by $15 \mathrm{~mm} \mathrm{Hg}$ and interstitial pressure by $12 \mathrm{~mm} \mathrm{Hg}$ (point $\mathrm{C}$ in Fig. 6), distal tubular diameter would be as sensitive an indicator of changes in interstitial pressure as it was at point $\mathrm{A}^{\prime}$ (when tubular pressure was increased by $5 \mathrm{~mm}$ $\mathrm{Hg}$ and interstitial pressure by $2 \mathrm{~mm} \mathrm{Hg}$ ). In each instance the estimate of the increase in interstitial pressure should be accurate to within $\pm 1 \mathrm{~mm} \mathrm{Hg}$.

Estimate of the change in interstitial pressure during infusion of furosemide or mannitol. In subsequent experiments, the behavior of the proximal and distal tubule during furosemide and mannitol diuresis was examined in terms of the theoretical framework described above. During furosemide diuresis, $\Delta D / \Delta P$ for both the proximal and distal tubule (Figs. 3 and 4) was indistinguishable from tubular compliance, suggesting that interstitial pressure did not increase. Since the increase in tubular pressure was small (less than $7 \mathrm{~mm} \mathrm{Hg}$ ) in approximately half the furosemide studies, we can assume (given the 95\% confidence intervals around the distal tubular diameter measurements), that if any increase in interstitial pressure occurred, it must have been less than $2 \mathrm{~mm} \mathrm{Hg}$. In those furosemide studies in which the increase in tubular pressure was larger $(7-15$ $\mathrm{mm} \mathrm{Hg}$ ) our estimate is less precise, but analysis of the proximal tubular data in these studies suggest that if any change in interstitial pressure occurred, it must have been less than $5 \mathrm{~mm} \mathrm{Hg}$.

It is obvious, of course, that the interpretation that interstitial pressure was unchanged during furosemide diuresis (an interpretation based on the agreement between compliance and the furosemide pressure-diameter curves) is valid only if we assume that tubular compliance was not altered by the furosemide. This assumption seems reasonable since agreement between the curves could have occurred in the face of a significant change in interstitial pressure only if two highly unlikely conditions were satisfied. First, furosemide would have had to alter both proximal and distal tubular compliance in a fashion so as to nearly exactly counterbalance the change in interstitial pressure; such behavior seems extremely improbable in view of the very different structure and different compliances of these two portions of the tubule. Second, the effect of furosemide upon tubular compliance would have had to have been independent of dosage since a fivefold increase in the quantity of furosemide administered had no notable influence on the response of tubular diameter.

The pressure-diameter relationships during mannitol diuresis were, in contrast to furosemide, consistent with a considerable change in interstitial pressure (Figs. 3 and 4). As shown in Table I, in the studies with mannitol in which tubular pressure was increased by 3-8 $\mathrm{mm} \mathrm{Hg}$, distal tubular diameter changes were consistent with a concomitant increase in interstitial pressure of 3-5 $\mathrm{mm} \mathrm{Hg}$ : in the studies in which tubular pressure was increased by $12-15 \mathrm{~mm} \mathrm{Hg}$, distal diameter changes were consistent with a concomitant increase in interstitial pressure of $7-10 \mathrm{~mm} \mathrm{Hg}$. It is noteworthy that in both the low-pressure and high-pressure experiments, the estimated increase in interstitial pressure was approximately $70 \%$ of the increase in tubular pressure. The data from the proximal tubule, although much less precise, indicate a rise in interstitial pressure of similar magnitude (Table I).

With mannitol administration, as in the case of furosemide, it seems highly improbable that the results were significantly influenced by an alteration in tubular compliance. To account for the experimental findings by a change in compliance, one would have to make the unlikely postulation that mannitol at each dosage level altered both proximal and distal tubular compliance in such a way as to fortuitously mimic a uniform and proportionate increase in interstitial pressure. ${ }^{8}$

A comparison of estimated collecting-duct resistance in the two diuretic states also strongly suggests that interstitial pressure increased more during mannitol than during furosemide administration. Resistance to flow through the collecting ducts $(R)$ can be calculated from the equation, $R=\Delta P / F$, where $\Delta P$ is the pressure gradient across the collecting ducts and $F$ is the flow through the collecting ducts. The pressure gradient across the collecting ducts $(\Delta P)$ can be taken as equal to distal pressure since pelvic pressure is less than 1 $\mathrm{mm} \mathrm{Hg} \mathrm{(3).} \mathrm{Flow} \mathrm{through} \mathrm{the} \mathrm{collecting} \mathrm{ducts}(F)$

\footnotetext{
${ }^{8}$ The conclusion that interstitial pressure is increased during mannitol diuresis is supported by the observation that renal subcapsular pressure in the dog increases markedly during mannitol administration (8).
} 
during the high-dose diuretic periods can be taken as equal to urine flow given that, at the observed high rates of urine flow (greater than $10 \%$ of GFR), collecting duct reabsorption is negligible. When the equation is solved using these values, collecting-duct resistence is found to be almost $50 \%$ greater during mannitol than during furosemide diuresis. This difference in behavior indicates that collecting-duct diameter increased less and (assuming no drug-induced changes in compliance) that interstitial pressure increased more with mannitol than with furosemide administration.

A similar comparison of the estimated resistance to flow through the loop of Henle in the two diuretic states also provides support for the interpretation that interstitial pressure increased more with mannitol diuresis than with furosemide diuresis. However, we are less confident of this interpretation than in the case of the collecting duct, since our estimate of flow through the loop is much less precise. As shown in Fig. 5, the pressure gradient across the loop of Henle decreased significantly as distal pressure increased during furosemide diuresis but not during mannitol diuresis. If one assumes that during administration of each drug proximal tubular water reabsorption was decreased by an approximately equal amount $(9,10)$ and that loop water reabsorption was roughly the same, we can reasonably conclude that loop resistance was significantly greater (and interstitial pressure higher) during mannitol than during furosemide administration. In fact, even if the increase in loop flow during furosemide diuresis was only half as large as during mannitol diuresis, we would still be forced to the interpretation that loop resistance and interstitial pressure were greater during mannitol administration.

Finally, we should point out that the compliance characteristics of the tubules, through their influence on cellular configuration, may well play a significant role in determining ionic permeability at various tubular and peritubular pressures. Boulpaep (1) has recently suggested that changes in the permeability of the proximal tubule to sodium and raffinose during saline diuresis are mediated through changes in the size and shape of the lateral intercellular spaces, a view supported by anatomical studies both in Necturus and in the rat $(11,12)$. Similar changes in the permeability characteristics of the proximal and distal tubule have been observed by other investigators in a variety of settings associated with increased tubular and peritubular pressure (13, 14). Given this background, it seems reasonable to suggest that a systematic examination of the interrelationships between tubular compliance, tubular anatomy, and transepithelial ionic permeability should lead to greater insight into the control of net ion movement.

\section{ACKNOWLEDGMENT}

This work was supported in part by Grant HE759 from the National Heart and Lung Institute, National Institutes of Health.

\section{REFERENCES}

1. Boulpaep, E. L. 1972. Permeability changes of the proximal tubule of Necturus during saline loading. Am. J. Phy'siol. 222 : 517.

2. Welling, L. W., and J. J. Grantham. 1972. Physical properties of isolated perfused renal tubules and tubular basement membranes. J. Clin. Invest. 51: 1063.

3. Cortell, S., M. Davidman, F. J. Gennari, and W. B. Schwartz. 1972. Catheter size as a determinant of outflow resistance and intrarenal pressure. Am. J. Physiol. 223: 910 .

4. Steinhausen, M. 1963. Eine methode zur differenzierung proximaler und distaler tubuli der nierenrinde von ratten in vivo und ihre anwendung zur bestimmung tubulärer strömungsgeschwindigkeiten. Arch. Gesamte. Physiol. Menschen Tiere (Pfluegers). 277: 23.

5. Gottschalk, C. W., and M. Mylle. 1956. Micropuncture study of pressures in proximal tubules and peritubular capillaries of the rat kidney and their relation to ureteral and renal venous pressures. Am. J. Phy'siol. 185: 430.

6. Gennari, F. J., S. Cortell, and W. B. Schwartz. 1970. Loss of measured activity of inulin- $\mathrm{C}^{\mathbf{1 4}}$ and a corrective technique. J. Appl. Physiol. 28: 105.

7. Snedecor, G. W., and W. G. Cochran. 1967. Statistical Methods. Iowa State University Press, Ames, Iowa. 6th edition. 157.

8. Ott, C. E., L. G. Navar, and A. C. Guyton. 1971. Pressures in static and dynamic states from capsules implanted in the kidney. Am. J. Physiol. 221: 394.

9. Seely, J. F., and J. H. Dirks. 1969. Micropuncture study of hypertonic mannitol diuresis in the proximal and distal tubule of the dog kidney. J. Clin. Invest. 48: 2330.

10. Brenner, B. M., R. I. Keimowitz, F. S. Wright, and R. W. Berliner. 1969. An inhibitory effect of furosemide on sodium reabsorption by the proximal tubule of the rat nephron. J: Clin. Invest. 48: 290.

11. Whittembury, G., F. A. Rawlins, M. Perez-Gonzales, and E. L. Boulpaep. 1972. Paracellular pathway for ion flow in kidney tubule. Abstracts from the 5th International Congress of Nephrology, Mexico City. 804.

12. Caulfield, J. B., and B. F. Trump. 1962. Correlation of ultrastructure with function in the rat kidney. $A m$. J. Pathol. 40: 199.

13. Lorentz, W. B., Jr., W. E. Lassiter, and C. W. Gottschalk. 1972. Renal tubular permeability during increased intrarenal pressure. J. Clin. Invest. 51: 484.

14. Bank, N., W. E. Yarger, and H. S. Aynedjian. 1971. A microperfusion study of sucrose movement across the rat proximal tubule during renal vein constriction. $J$. Clin. Invest. 50: 294. 